\title{
DYNAMIC 3D MODELING OF A CANAL-TUNNEL USING PHOTOGRAMMETRIC AND BATHYMETRIC DATA
}

\author{
E. Moisan ${ }^{\mathrm{a}}$, C. Heinkele ${ }^{\mathrm{a}}$, P. Charbonnier ${ }^{\mathrm{a}}$, P. Foucher ${ }^{\mathrm{a}}$, P. Grussenmeyer ${ }^{\mathrm{b}}$, S. Guillemin ${ }^{\mathrm{b}}$, M. Koehl ${ }^{\mathrm{b}}$ \\ ${ }^{a}$ Cerema Est, Laboratoire de Strasbourg, 11 rue Jean Mentelin, B.P. 9, 67035 Strasbourg, France \\ (Emmanuel.Moisan, Christophe.Heinkele, Pierre.Charbonnier, Philippe.Foucher)@cerema.fr \\ ${ }^{\mathrm{b}}$ ICube Laboratory UMR 7357, Photogrammetry and Geomatics Group, INSA Strasbourg, 24 Boulevard de la Victoire, \\ 67084 Strasbourg, France - (pierre.grussenmeyer, samuel.guillemin, mathieu.koehl)@insa-strasbourg.fr
}

\section{Commission II}

KEY WORDS: 3D modeling, multibeam echosounder, photogrammetry, inspection, underwater

\begin{abstract}
:
This contribution introduces an original method for dynamically surveying the vault and underwater parts of a canal-tunnel for 3D modeling. The recording system, embedded on a barge, is composed of cameras that provide images of the above-water part of the tunnel, and a sonar that acquires underwater 3D profiles. In this contribution we propose to fully exploit the capacities of photogrammetry to deal with the issue of geo-referencing data in the absence of global positioning system (GPS) data. More specifically, we use it both for reconstructing the vault and side walls of the tunnel in 3D and for estimating the trajectory of the boat, which is necessary to rearrange sonar profiles to form the 3D model of the canal. We report on a first experimentation carried out inside a canal-tunnel and show promising preliminary results that illustrate the potentialities of the proposed approach.
\end{abstract}

\section{INTRODUCTION}

In order to enable inland navigation, 33 canal-tunnels were bored during the 19th and 20th centuries in France. Nowadays, most of them are still in use, for commercial navigation and pleasure boating. Thus, maintaining them meets a double need: preserving a heritage and ensuring safety. To reach this goal, periodical visual inspections are necessary, which is both time-consuming and impeding for traffic. Moreover, surveying the underwater parts of tunnels by divers is rather awkward due to the turbidity of water and tunnel drying may be hazardous for the tunnel structure. Finally, traditional bathymetric techniques cannot be employed due to the lack of Global Navigation Satellite Systems (GNSS) signal in tunnels. Therefore, innovative solutions are needed.

In this context, we are developing an automatic inspection system, in collaboration with Voies Navigables de France (VNF, the French operator of waterways) and the Centre d'Études des TUnnels (CETU). In this paper, we introduce a method for surveying the above and underwater parts of the tunnel in a dynamic fashion and then, for reconstructing the full 3D model of the canal-tunnel.

The proposed prototype, which is embedded on a barge, associates image acquisitions for modeling the vaults to sonar recording, for the canal. Reconstructing the above-water parts in $3 \mathrm{D}$ from images is not very difficult thanks to photogrammetric techniques. The choice of sonar technology for surveying the canal is motivated by the turbidity of the water. A multibeam echosounder records $3 \mathrm{D}$ profiles of the canal walls and soil perpendicularly to its trajectory. Hence, it is necessary to rearrange these profiles along the recorded path of the boat to build the underwater part of the model. Since no GNSS signal is available in tunnels, we propose to estimate the trajectory thanks to the successive positions of the cameras provided by photogrammetry. In other words, we fully exploit the potential of photogrammetric techniques to replace the traditional GNSS-INS (Inertial Navigation Systems) couple. Of course, an evaluation of the method

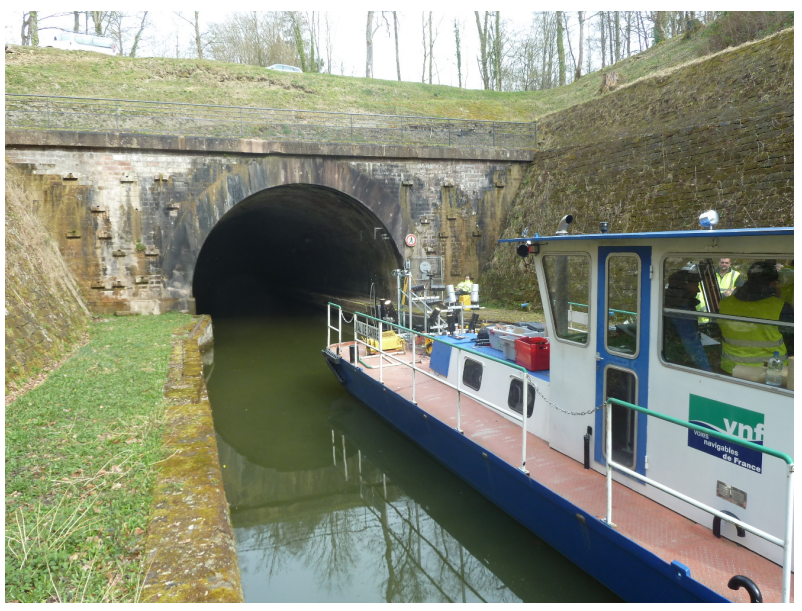

Figure 1. View of the barge supporting the modular acquisition system, on the experimental site.

will be needed, which can be done either by assessing the obtained trajectory using an external reference, or by comparing the reconstructed model to a reference one, built from static acquisitions (Moisan et al., 2015), as described in (Charbonnier et al., 2013).

In this paper, we report on a first experimental campaign, that was performed in a canal-tunnel near Strasbourg (France), and show the first 3D models obtained with the proposed method.

The rest of the paper is organized as follows. First a brief overview of related works is provided in Sect. 2. In Sect. 3, we introduce the acquisition set-up and describe the acquisition procedure. Then, Sec. 4 presents the proposed processing method and the experimental results. Finally, Sec. 5 concludes the paper and proposes future directions for this work. 


\section{RELATED WORK}

Recently, several systems were devised for surveying 3D semisubmerged structures such as harbours or dams, e.g. (Rondeau et al., 2012). These systems are based on the combination of terrestrial mobile mapping and bathymetry. They associate a Terrestrial Laser Scanner (TLS), for surveying above-water parts, and a multibeam echosounder, for the underwater ones. Since the dynamic use of these two devices requires the position and orientation of the acquisition platform during surveying, INS and GNSS are employed in open environments. However, the lack of GNSS signal inside tunnels makes this localisation method inoperative in our context.

An alternative, proposed in (Papadopoulos et al., 2014), is based on high-update-rate TLS acquisitions: the trajectory is estimated by registering scans with each other thanks to the ICP (Iterative Closest Point) algorithm. To be effective, this method requires common features between scans, which might be difficult to find in tubular environments, such as tunnels. Moreover, such methods often use rotative scanners and it is then necessary to carefully eliminate rotational effects in the scans to avoid false correspondences and hence to estimate the trajectory properly.

Photogrammetry is an appealing solution since it enables gathering navigation information at the same time as reconstructing the $3 \mathrm{D}$ model. This technique can be used in real-time, which is called visual odometry. It was chosen in (Kriechbaumer et al., 2015) to automatically survey river banks from a moving vessel. Photogrammetry may also be employed off-line, using bundle adjustment, as in (Charbonnier et al., 2013), for example: the trajectory is then derived from the successive camera positions and orientations. This is the approach we choose for this paper.

\section{DATA RECORDING}

To experiment our method, a prototype was developed and tested in a tunnel-canal. The system includes a set of cameras that record images of the vault and side walls, and an underwater sensor to survey the canal. All these sensors were placed on a boat thanks to a modular structure (Charbonnier et al., 2014), shown on Fig. 2.

\subsection{Set-up}

The image acquisition system is made of six industrial cameras and one photographic camera. In fact, four cameras are arranged to form two stereo rigs. The first one is directed towards the vault, perpendicularly to the tunnel longitudinal axis (green cameras, called cam5 and cam6, on Fig. 2). Since the aperture angle of the cameras does not allow capturing all the vault, several passes are needed, with two different angles with respect to the vertical axis of the tunnel $\left(58^{\circ}\right.$ and $\left.0^{\circ}\right)$, as shown on Fig. 3. The second one is horizontal and placed so as to take pictures of the right side wall, parallel to it (blue cameras, cam 1 and cam2, on Fig. 2). The remaining two cameras are also used to take pictures of the side wall, but with an oblique angle, which is necessary to limit curvature effects in the reconstructed model (see red cameras, cam3 and cam4, on Fig. 2), as shown in (Nocerino et al., 2014). These six cameras are Pike F210 C $(1920 \times 1080$ pixels $)$ cameras with $12.5 \mathrm{~mm}$ focal length. They are triggered simultaneously, at a $5 \mathrm{~Hz}$ frequency. The industrial cameras are individually calibrated with well-known procedure (Tsai, 1987, Zhang, 1999, Szeliski, 2010) using standard calibration patterns. Stereo rigs are also calibrated in order to measure in 3D.
The photographic camera is a Canon EOS 5D MarkIII $(5760 \times$ 3840 pixels) with a fisheye lens. It takes wide-angle pictures, triggered every $2 \mathrm{~s}$, that may be used to ease $3 \mathrm{D}$ reconstruction. All these elements are attached to the modular structure, that also supports lightings, and is placed on the front deck of the barge.

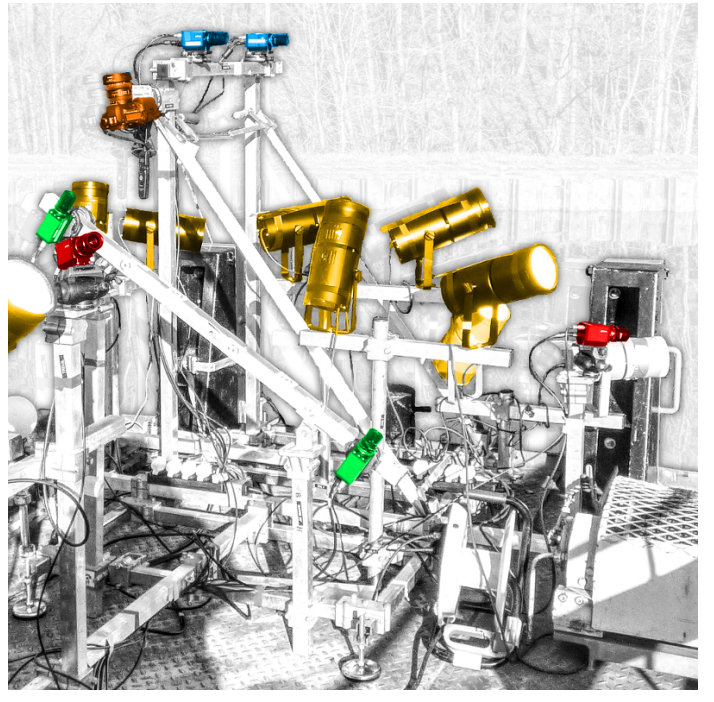

Figure 2. Image acquisition system with the first stereo rig in green, the second stereo-rig in blue, the oblique cameras in red, the fish-eye camera in orange, and the lighting system in yellow.

The sonar device used for underwater 3D data acquisition is a multibeam echosounder, namely the MB1350 (Blueview). For this first experimentation the device was operated by a subcontractor, the Sub-C Marine company (France). The device is attached to the boat thanks to a removable pole. The emitted sound wave has a frequency of $1.35 \mathrm{MHz}$ and the receiving antenna is composed of 256 beams arranged in a fan shape with $45^{\circ}$ aperture. It acquires point profiles at a regular rate of $40 \mathrm{~Hz}$. The high frequency of the emitted signal offers a good resolution in distance, but at the same time, limits the acquisition range to $30 \mathrm{~m}$. Moreover, due to the $1^{\circ}$ beam width, the signal footprint on a perpendicular plane $5 \mathrm{~m}$ away from the device is a square of $87 \mathrm{~mm}$ side. More information about the sonar capacities may be found in (Moisan et al., 2016), where it was used to survey a lock in a static manner thanks to a mechanical rotation system. Several observations have been made, such as the granular aspect of the resulting point cloud or the presence of acoustic phenomena or, the ability to detect defects of decimetric size. In dynamic acquisitions, the surveyed area is swept thanks to the vessel motion and the $3 \mathrm{D}$ model is obtained by gathering profiles along the trajectory of the system. However, due to the limited aperture angle, several passes are necessary. In this experiment, three tilt angles were applied to the MB1350: $11.25^{\circ}, 45^{\circ}$ and $90^{\circ}$ (see Fig. 3). Regarding sonar calibration, only the speed of sound in water was measured with a suitable instrument.

\subsection{Experimentation}

The experimental site is the canal-tunnel of Niderviller (Lorraine region, France), see Fig. 1. This underground waterway was built between 1839 and 1845 on the Marne-Rhine canal. It is straight, $475 \mathrm{~m}$ long and has a pedestrian path on a ledge. A set of reference points, known in the French reference system (RGF93 and NGF-IGN 69) was implanted inside the tunnel. In particular, reflective plates were placed every decameter on the sidewall 

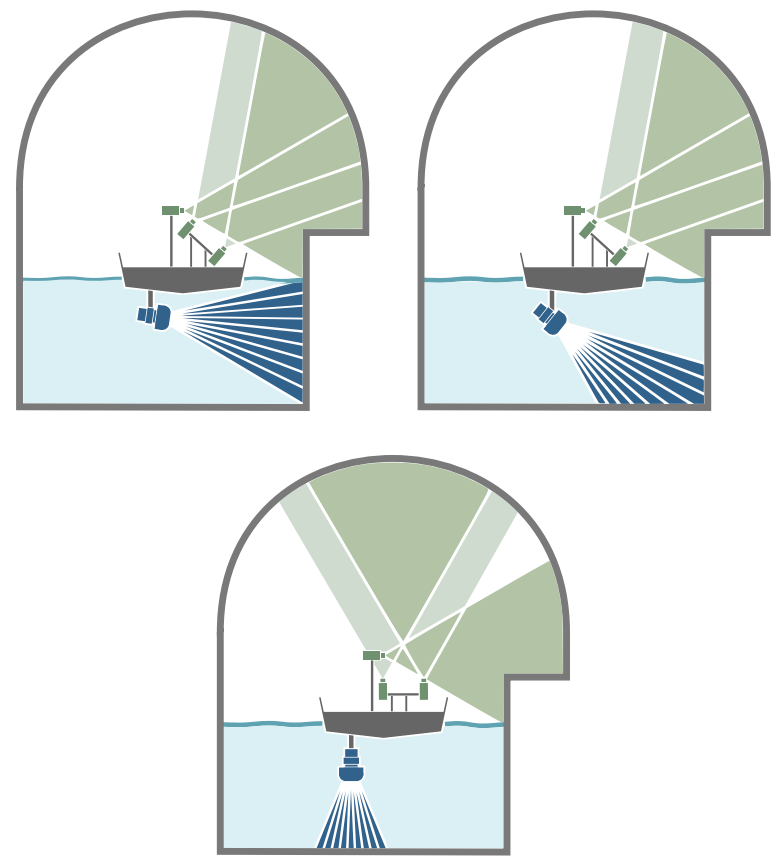

Figure 3. Schematic views of the three system configurations used for scanning a half tunnel.

along the bench. A well-suited lighting makes these landmarks easily detectable in images using simple image processing algorithms. In the photogrammetric process, they help scaling and geo-referencing the model.

As previously mentioned, three different set-ups of the sonar and two stereo-rig orientations are required to enable the survey of the half vault and canal. Thus, a minimum of three back and forth passes inside the tunnel are necessary (see Fig. 3). As the boat speed was roughly $1 \mathrm{~m} . \mathrm{s}^{-1}$, images are taken every $20 \mathrm{~cm}$ and sonar profiles, every $2.5 \mathrm{~cm}$. Hence, during each pass through the tunnel, about 15000 images are recorded by industrial cameras, and almost 20000 profiles are acquired by the sonar. Last, roughly 250 pictures are taken by the photographic camera.

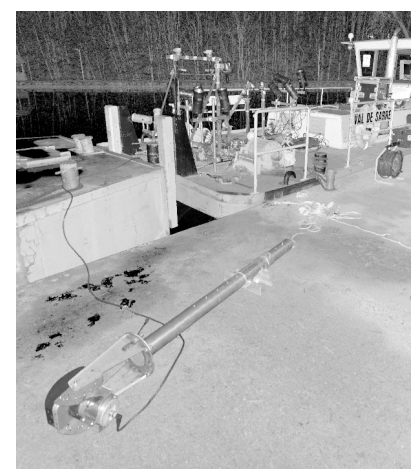

Figure 4. Intensity image taken while scanning the sonar and its supporting mast (in the foreground). The imaging system can be seen in the background.

Furthermore, in order to estimate the relative position between each sensor, namely the lever-arm (see $\$ 4.1$ ), each set-up has to be recorded. Therefore, the whole system was surveyed thanks to a Terrestrial Laser Scanner (TLS) each time an element was modified (stereo rig orientation or sonar orientation). Scans were carried out on the boat for all devices attached to the modular structure, and from the dock for above-water part of the sonar support. The sonar and its mast were also scanned onshore, as shown on Fig. 4.

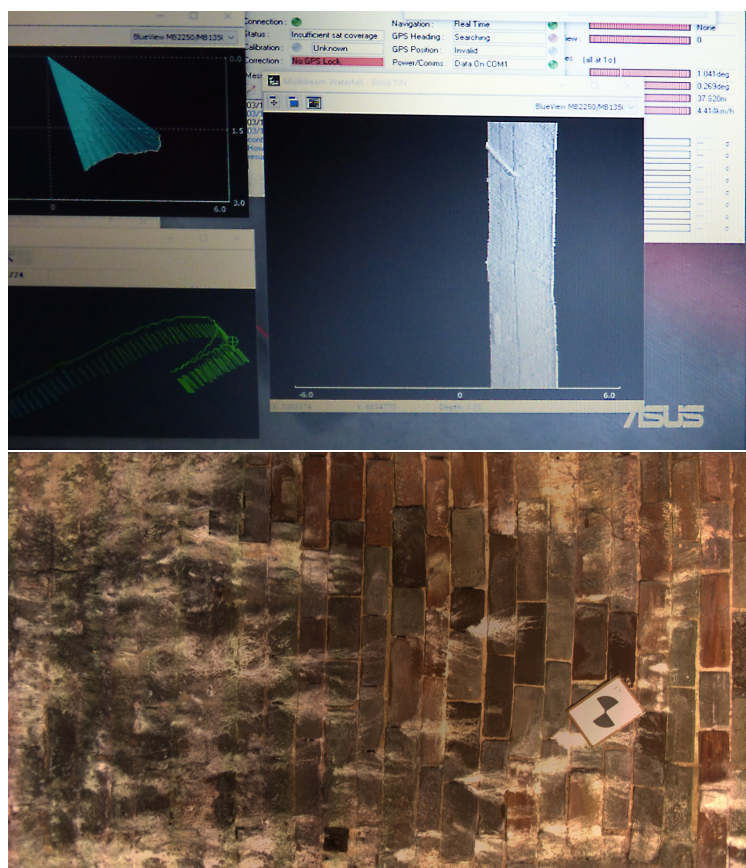

Figure 5. Views of sonar acquisitions during the experimentation (top) ; typical image of the vault (bottom).

Finally, in order to foresee the method evaluation, the boat motion was recorded using tacheometric tracking, thanks to a couple of active prisms attached to the cabin of the boat.

\section{3D MODELING}

At the end of the acquisition campaign, we have in hand several image sequences of the side walls and vaults, and several series of sonar profiles (see Fig. 5). To construct the full 3D model of the canal-tunnel with these data in the absence of GPS signal, we propose a method that fully exploits the capacities of photogrammetry.

Using photogrammetry to build the above-water model is rather straightforward thanks to bundle adjustment techniques (Luhmann et al., 2014). A simplified visual odometry method (Charbonnier et al., 2014) is applied, which allows tiling the images (i.e. gathering them by cross-sections) along the tunnel in order to reduce the computational requirements of the correspondence matching. Images are processed using photogrammetric software MICMAC APERO (Pierrot-Deseilligny and Clery, 2011). A bundle adjustment is performed, taking into account rigidity constraints between the six cameras at each acquisition time. This step also provides the successive positions $\mathbf{P}_{t i}^{n}$ and orientations of the six cameras in a common geo-referenced coordinate system.

Reconstructing the 3D model of the canal requires rearranging the sonar profiles along its trajectory. The latter may be estimated by registering a model of the image acquisition system on the 
set of successive camera positions provided by the bundle adjustment. To this end, it is necessary to estimate the relative position of all sensors, which is called lever-arm estimation.

In the following, we first describe the level-arm estimation process and then, we introduce the 3D sonar model reconstruction.

The notations used in this section are summarized in Tab. 1. In particular, sonar data are given in a 2D polar coordinates system centered at the sonar acquisition center, whose axes are given by the central beam of the swath and its perpendicular direction in the swath plane (see Fig. 6).

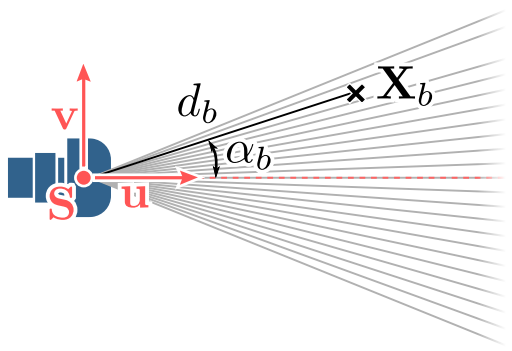

Figure 6. Definition of the coordinate system and measurement vector attached to the sonar.

\begin{tabular}{|c|l|}
\hline $\mathbf{X}$ & Acquired sonar point \\
\hline $\mathbf{S}$ & Sonar acquisition center \\
$\mathbf{u}$ & Direction vector of the central sonar beam \\
$\mathbf{v}$ & Vector in the sonar swath plane and perpendicular \\
& to $\mathbf{u}$ \\
\hline$d$ & distance from $\mathbf{X}$ to $\mathbf{S}$ \\
$\alpha$ & Beam angle \\
\hline $\mathbf{P}$ & Optical centers of industrial cameras \\
\hline $\mathbf{R}$ & Rotation matrix \\
$\mathbf{T}$ & Translation vector \\
\hline \hline$\ldots v$ & Element defined in the vessel coordinate system \\
\hline$\cdots$ & Element defined in the national coordinate system \\
\hline \hline$\cdots t i$ & Element defined for the $t i$ images acquisition \\
$\cdots t s$ & Element defined for the $t s$ sonar acquisition \\
\hline$\cdots b$ & Element defined for one beam of the sonar'swath \\
\hline
\end{tabular}

Table 1. Chart of the notations used in this section.

\subsection{Lever-arm estimation}

Geo-referencing the sonar profiles requires estimating the relative offsets of sensors (lever-arm), i.e. calibrating the system. We carry out a direct estimation using a global point cloud of the experimental setup built from the TLS survey of the imaging system, shown in red on Fig. 7, and TLS surveys of the sonar and its supporting mast, shown in blue (indeed, an acquisition is performed each time the tilt angle of the sonar is modified, as noticed in $\S 3.2$ ).

To align both models, features on the supporting mast are used, namely the axis, handles and top of the tube. The alignment procedure provides a $3 \mathrm{D}$ point cloud of the whole acquisition system.

In a second step, we align the Computed-Aided-Design (CAD) model of each sensor on the point cloud, to obtain their accurate position and orientation. More specifically, we first segment the

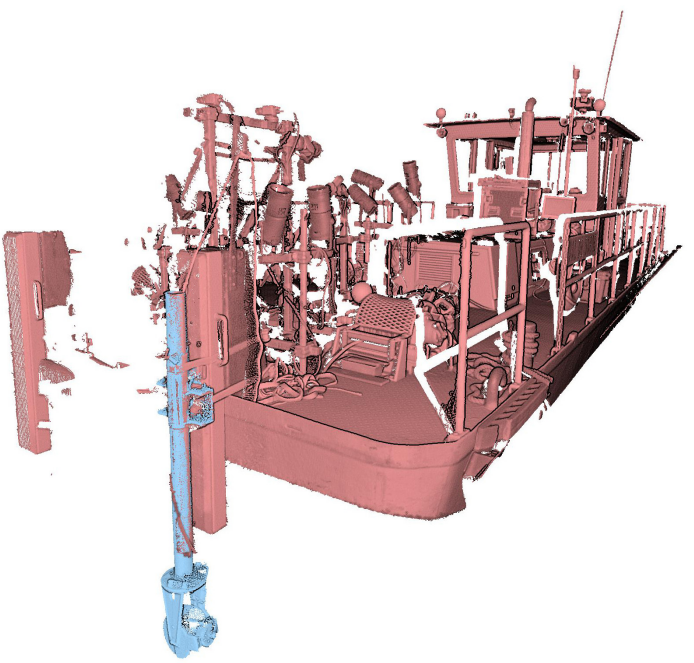

Figure 7. Full-set-up point cloud obtained by laser scanning with in red the above-water part of the system and in blue the aligned sonar point cloud.
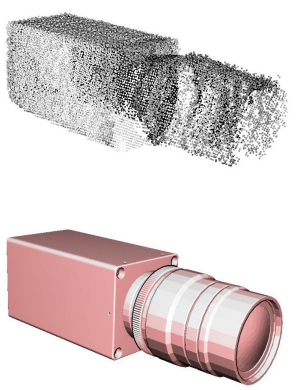

Figure 8. Segmented point cloud exemple of an industrial camera from TLS acquisitions (top) and the corresponding CAD constructor model (bottom).

global point cloud to isolate each sensor (see Fig. 8, top) then we align the CAD model (see Fig. 8, bottom) on it.

To perform mesh-to-point cloud alignement, an ICP-like algorithm was developed. A first modification to the initial algorithm concerns the mesh-point cloud distances computation: we apply the method introduced in (Bærentzen and Aanæs, 2002). Moreover, at each iteration of the ICP algorithm, the new transformation is estimated from vectors defined by points of the cloud and their normal projections on the mesh.

Since the CAD model are furnished by the constructors of the sensors, their acquisition centers are accurately located. Hence, at the end of the procedure, we know the optical center position of the cameras, $\mathbf{P}^{v}$, and the position and orientation of the echosounder measuring head, in a common coordinate system (i.e. $\mathbf{S}^{v}, \mathbf{u}^{v}$ and $\mathbf{v}^{v}$ ) attached to the vessel.

Notice that the accurate position of the prism centers may be extracted from the global point cloud using a constructor CAD model as well.

\subsection{D sonar point cloud building}

Now, we introduce the applied process to reconstruct the underwater model from sonar acquisitions. The process follows three 


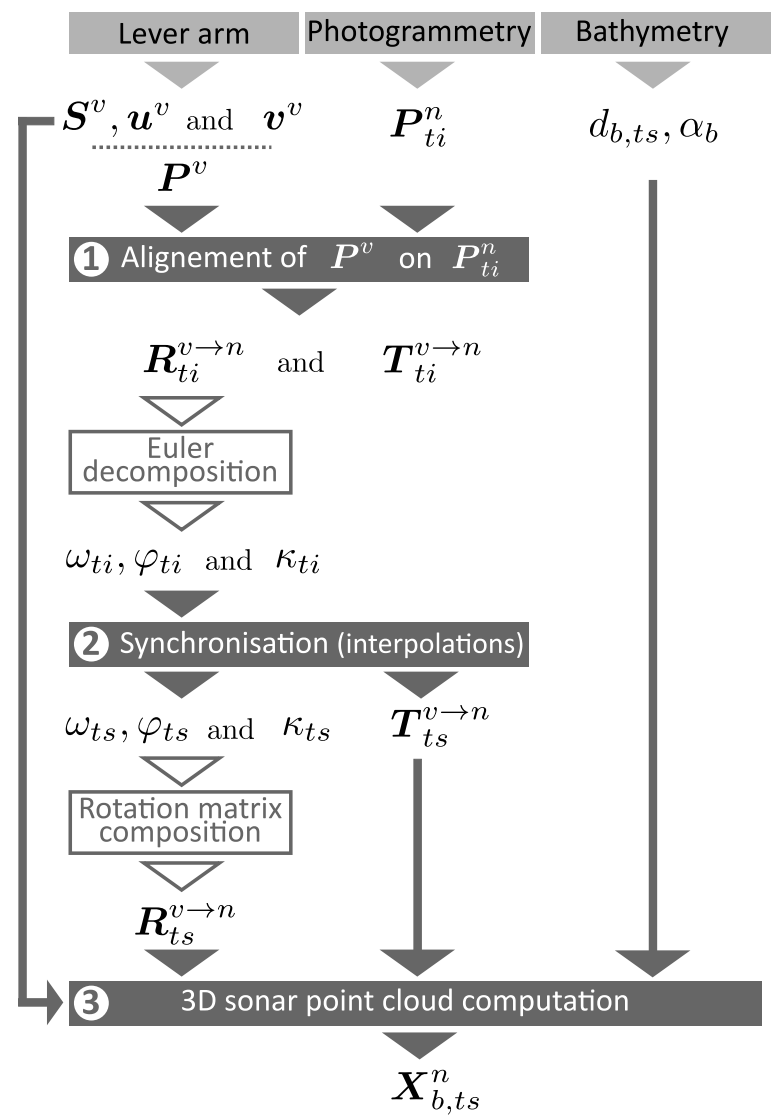

Figure 9. Diagram of sonar point cloud process. Notations are introduced in Tab. 1.

major steps, summarized on Fig. 9. At the first time, the positions of the camera optical centers defined in the vessel coordinates system are transformed into the national coordinate one. At each image acquisition, positions $\mathbf{P}^{v}$ provided by the lever-arm are aligned on photogrammetric computed positions $\mathbf{P}_{t i}^{n}$. The Procrustes method, described in (Golub and Van Loan, 2012), is applied to estimate both the rotation $\left(\mathbf{R}_{t i}^{v \rightarrow n}\right)$ and translation $\left(\mathbf{T}_{t i}^{v \rightarrow n}\right)$ matrices. Note that the rotation matrix is decomposed into Euler angles $\omega_{t i}, \varphi_{t i}, \kappa_{t i}$.

In the second major step, sonar and image acquisitions are synchronized. At each sonar acquisition, translation vector $\left(\mathbf{T}_{t s}^{v \rightarrow n}\right)$ and rotation matrix $\left(\mathbf{R}_{t s}^{v \rightarrow n}\right)$ need to be estimated. The translation vector is computed by linear interpolation. Indeed, we assume that the trajectory is roughly linear and the boat speed between two camera acquisitions is constant. The rotation matrix is given by the Euler angles $\omega_{t s}, \varphi_{t s}, \kappa_{t s}$. These angles are computed by using spline interpolations from Euler angles $\omega_{t i}, \varphi_{t i}, \kappa_{t i}$.

Lastly, every sonar profile is geo-referenced by applying the previously computed transformations. This operation begins by computing beam direction vectors for each acquisition. Then, we straightforwardly obtain the sonar point cloud $\mathbf{X}_{b, t s}^{n}$ in the national reference system.

\subsection{Results}

We first study the inter-camera distances given by the direct estimation described in $\S 4.1$, the bundle adjustment and the stereo calibration, shown in Tab. 2. We note that the distances obtained by photogrammetry are mean distances. Indeed, despite the rigidity constraints, we observed small variations, especially at the extremities of the image sequences. However, for the stereo rigs (cam1-cam2 and cam5-cam6), the difference between photogrammetry and stereo calibration is small: about 1 or $2 \mathrm{~mm}$. One may observe that the difference between TLS survey ( $\S 4.1$ ) and photogrammetry ranges from $1 \mathrm{~mm}$ to $3.7 \mathrm{~cm}$. These differences include errors from both methods. However, we believe that the photogrammetric processing can be improved.

\begin{tabular}{|c||c||c|c||c|c|}
\cline { 2 - 6 } \multicolumn{1}{c|}{} & \multicolumn{1}{c||}{ TLS } & \multicolumn{2}{c||}{ photogrammetry } & \multicolumn{2}{c|}{ stereo calibration } \\
\cline { 2 - 6 } \multicolumn{1}{c|}{} & dist. & $\begin{array}{c}\text { mean } \\
\text { dist. }\end{array}$ & diff. & dist. & diff. \\
\hline cam1-cam2 & 0.499 & 0.503 & -0.004 & 0.502 & -0.003 \\
cam1-cam3 & 1.556 & 1.536 & 0.020 & - & - \\
cam2-cam3 & 2.027 & 2.009 & 0.018 & - & - \\
cam1-cam4 & 1.472 & 1.496 & -0.023 & - & - \\
cam2-cam4 & 1.589 & 1.626 & -0.037 & - & - \\
cam3-cam4 & 1.617 & 1.601 & 0.017 & - & - \\
cam1-cam5 & 1.591 & 1.598 & -0.007 & - & - \\
cam2-cam5 & 1.904 & 1.908 & -0.005 & - & - \\
cam3-cam5 & 0.986 & 0.967 & 0.018 & - & - \\
cam4-cam5 & 0.984 & 0.985 & -0.001 & - & - \\
cam1-cam6 & 0.930 & 0.916 & 0.015 & - & - \\
cam2-cam6 & 1.390 & 1.372 & 0.018 & - & - \\
cam3-cam6 & 0.739 & 0.730 & 0.009 & - & - \\
cam4-cam6 & 1.472 & 1.478 & -0.007 & - & - \\
cam5-cam6 & 0.999 & 1.009 & -0.010 & 1.007 & -0.008 \\
\hline
\end{tabular}

Table 2. Distances between cameras (in $m$ ) obtained from lever-arm estimation, photogrammetry and optical calibration.

Differences (in $m$ ) between TLS distance and the two other computation methods are also given.

A rough model is shown on Fig 10. This model was built from simplified trajectories, i.e. we considered the boat motion as linear, at a constant speed, and the geo-referencing required manual processing. The resulting model offers a good visualisation underwater part of the tunnel, but this method leads to wrongly placed elements. Moreover, when merging the models provided by different acquisitions, one observes longitudinal shifts.

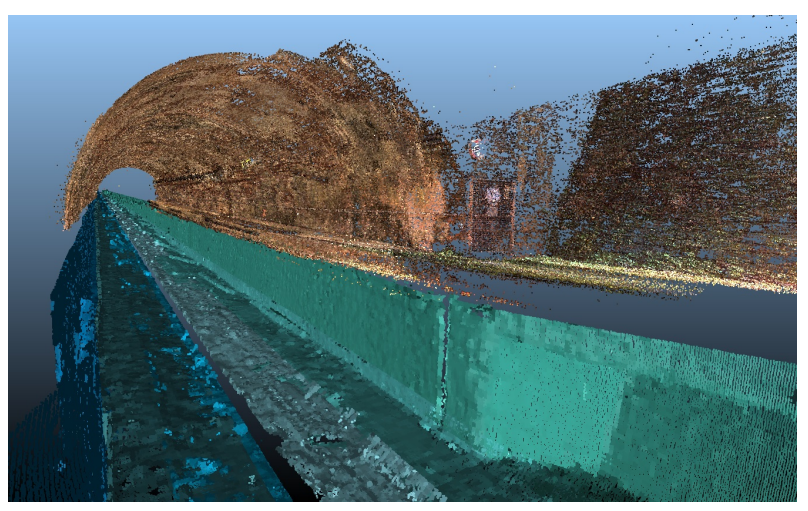

Figure 10. Rough model obtained from dynamic acquisitions and an approximated trajectory of the boat.

On the contrary, thanks to the trajectory estimation and compensation method described in $\S 4.2$, both photogrammetric and sonar models are straightforwardly referenced in a same geographic coordinate system. A preliminary result, obtained on a piece of tunnel is shown in Fig. 11. As can be seen, the alignement of the above-water and underwater point clouds is visually much more satisfying. 


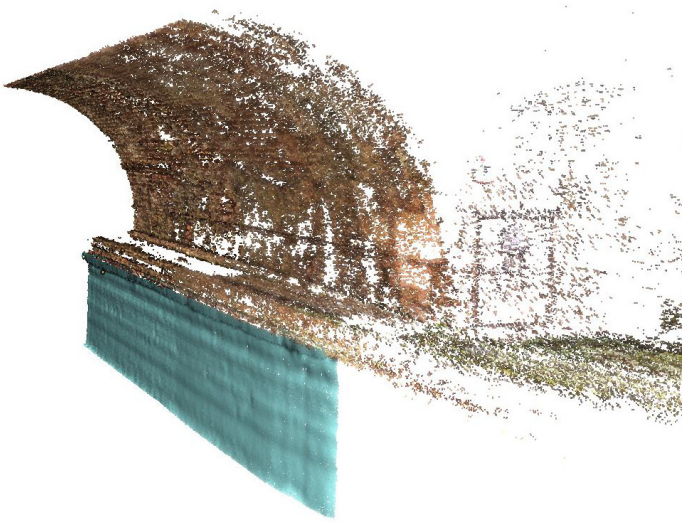

Figure 11. Part of the final model resulting from the developed approach.

However, two kinds of issues were highlighted during data processing. First, we observed imperfection in time synchronisation. Such errors can have a large impact on modeling since a shift of $0.01 \mathrm{~s}$ on time stamping cause a $1 \mathrm{~cm}$ error, bearing in mind that the boat speed is roughly $1 \mathrm{~m} \cdot \mathrm{s}^{-1}$. In order to improve it, adjustments on the prototype are requested. Second, the estimation over time of the system orientation has a significant impact on the resulting underwater model and still needs improvements.

\section{CONCLUSION AND FUTURE WORK}

In this paper, we have introduced a method to model a canaltunnel in $3 \mathrm{D}$ by using photogrammetric and bathymetric data. To reach this goal, we had to address an important issue in georeferencing bathymetric data, namely the lack of GNSS signal inside the tunnel. Photogrammetry was selected to build the abovewater model, since images suffice to compute a $3 \mathrm{D}$ point cloud, but also to estimate the trajectory of the boat, in order to georeference sonar data. We reported on the first experimentation of our system in a canal-tunnel and provided preliminary results that illustrate the potentialities of the method.

These encouraging results may be improved by perfecting the synchronisation between devices. Moreover, introducing temporal filtering may help robustifying the estimation and tracking of the orientation angles, leading to better trajectory estimations. Finally, the data gathered in several passes will be combined in a natural fashion by building a common photogrammetric model to estimate the whole set of trajectories simultaneously and register all sonar profile sequences to form a full 3D model of the canal.

In order to assess quantitatively the performance of the method, two kinds of experiments will be conducted in a near future. First, differences between the trajectory obtained by photogrammetry and the one measured by tacheometry will be analyzed. Second, a reference model (see Fig. 12), which was built from static TLS and sonar 3D acquisitions during a previous experiment (Moisan et al., 2015), will be used to assess the dynamic model, according to the methodology proposed in (Charbonnier et al., 2013).

\section{ACKNOWLEDGEMENTS}

This work was funded by the Cerema under PhD Grant No. 64 and supported by the Cerema research program APHOGEOPHY

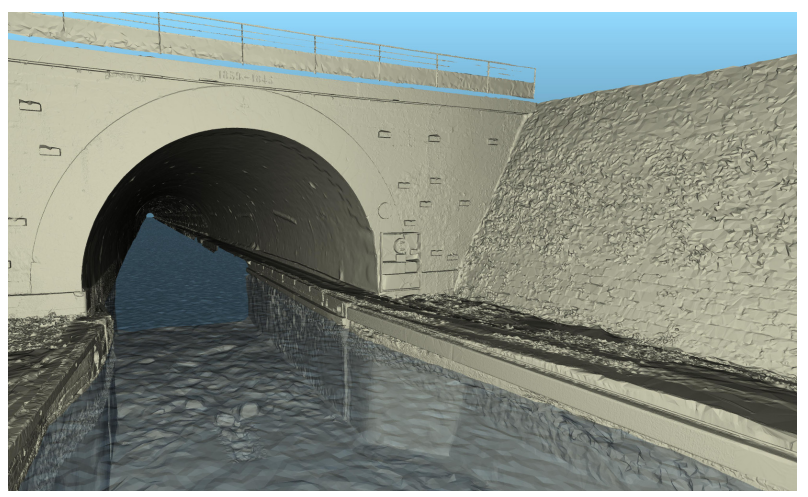

Figure 12. 3D reference model of Niderviller tunnel entrance.

funded by the General directorate for infrastructure, transport and the sea (DGITM, French Ministry of Environment, Energy and the Sea).

\section{REFERENCES}

Bærentzen, J. A. and Aanæs, H., 2002. Generating signed distance fields from triangle meshes. Informatics and Mathematical Modeling, Technical University of Denmark, DTU.

Charbonnier, P., Chavant, P., Foucher, P., Muzet, V., Prybyla, D., Perrin, T., Grussenmeyer, P. and Guillemin, S., 2013. Accuracy assessment of a canal-tunnel 3D model by comparing photogrammetry and laserscanning recording techniques. In: 24th CIPA Symposium, Vol. XL-5, Int. Arch. Photogramm. Remote Sens. Spatial Inf. Sci., pp. 171-176.

Charbonnier, P., Foucher, P., Chavant, P., Muzet, V., Prybyla, D., Perrin, T., Albert, J., Grussenmeyer, P., Guillemin, S. and Koehl, M., 2014. An image-based inspection system for canal-tunnel heritage. International Journal of Heritage in the Digital Era 3(1), pp. 197-214.

Golub, G. H. and Van Loan, C. F., 2012. Matrix computations. Johns Hopkins Studies in the Mathematical Sciences, Vol. 3, 4 edn, Johns Hopkins University Press.

Kriechbaumer, T., Blackburn, K., Breckon, T. P., Hamilton, O. and Rivas Casado, M., 2015. Quantitative evaluation of stereo visual odometry for autonomous vessel localisation in inland waterway sensing applications. Sensors 15(12), pp. 31869-31887. Special Issue Robotic Sensory Systems for Environment Protection and Conservation.

Luhmann, T., Robson, S., Kyle, S. and Boehm, J., 2014. Closerange photogrammetry and $3 D$ imaging. 2 nd edn, Walter de Gruyter.

Moisan, E., Charbonnier, P., Foucher, P., Grussenmeyer, P., Guillemin, S. and Koehl, M., 2015. Adjustment of sonar and laser acquisition data for building the 3D reference model of a canal tunnel. Sensors 15(12), pp. 31180-31204. Special Issue Sensors and Techniques for 3D Object Modeling in Underwater Environments.

Moisan, E., Charbonnier, P., Foucher, P., Grussenmeyer, P., Guillemin, S., Samat, O. and Pagès, C., 2016. Assessment of a static multibeam sonar scanner for 3D surveying in confined subaquatic environments. In: ISPRS - International Archives of the Photogrammetry, Remote Sensing and Spatial Information Sciences, Vol. XLI-B5, Prague, Czech Republic, pp. 541-548. 
Nocerino, E., Menna, F. and Remondino, F., 2014. Accuracy of typical photogrammetric networks in cultural heritage 3D modeling projects. ISPRS - International Archives of the Photogrammetry, Remote Sensing and Spatial Information Sciences XL-5, pp. $465-472$.

Papadopoulos, G., Kurniawati, H., Shariff, A. S. B. M., Wong, L. J. and Patrikalakis, N. M., 2014. Experiments on surface reconstruction for partially submerged marine structures. Journal of Field Robotics 31(2), pp. 225-244.

Pierrot-Deseilligny, M. and Clery, I., 2011. Apero, an open source bundle adjustment software for automatic calibration and orientation of set of images. 38(5), pp. 269-276.

Rondeau, M., Leblanc, E. and Garant, L., 2012. Dam infrastructure first inspection supported by an integrated multibeam echosounder MBES)/LiDAR system. In: Proceedings of the Canadian Dam Association annual conference, Saskatoon, Canada.

Szeliski, R., 2010. Computer Vision: Algorithms and Applications. 1st edn, Springer-Verlag New York, Inc., New York, NY, USA.

Tsai, R., 1987. A versatile camera calibration technique for high-accuracy 3D machine vision metrology using off-the-shelf tv cameras and lenses. IEEE Journal on Robotics and Automation 3(4), pp. 323-344.

Zhang, Z., 1999. Flexible camera calibration by viewing a plane from unknown orientations. In: Proceedings of the Seventh IEEE International Conference on Computer Vision (ICCV), Vol. 1, IEEE, Kerkyra, Corfu, Greece, pp. 666-673. 